\title{
Pre-surgical Multiparametric Assessment of Breast Lesions Using 3-Tesla Magnetic Resonance
}

\author{
HYNEK MIRKA $^{1,2}$, RADEK TUPY ${ }^{1}$, ANDREA NARSANSKA $^{3}$, ONDREJ HES ${ }^{4}$ and JIRI FERDA ${ }^{2}$ \\ Departments of ${ }^{1}$ Imaging Methods and ${ }^{3}$ Surgery, and ${ }^{4}$ Sikl's Department of Pathology, Medical School and \\ Teaching Hospital Pilsen, Charles University in Prague, Pilsen, Czech Republic; \\ ${ }^{2}$ Biomedical Centre, Faculty of Medicine in Pilsen, Charles University in Prague, Pilsen, Czech Republic
}

\begin{abstract}
Background/Aim: The aim of this study was to evaluate experience with multiparametric breast imaging on 3-Tesla magnetic resonance (3T-MRI) scanner using a dedicated 18-channel coil compared to histological findings in women after surgery. Materials and Methods: The study included 100 women with 105 Breast Imaging Reporting and Data System (BI-RADS) 4 to 6 lesions by mammography who were examined using 3T-MRI and subsequently underwent surgery. MRI included non-contrast T1, T2 and T2 short tau inversion recovery $(S T I R)$ sequences, diffusion-weighted imaging with apparent diffusion coeficient maps, postcontrast dynamic study and single-voxel MRI spectroscopy. The results were compared to those of histopathological examination. Results: A sensitivity of $98.68 \%$ was found for the whole population, with a specificity of $86.20 \%$. The most valuable findings were diffusion restriction with sensitivity of $90.79 \%$ and specificity of $89.66 \%$, and increased choline in the spectrum with sensitivity of $68.42 \%$ and specificity of $93.10 \%$. Evaluation of the enhancement curve had sensitivity of $45.05 \%$ and specificity of $72.41 \%$. In examination of lymph nodes, 3TMRI had sensitivity of $92.59 \%$ and specificity of $93.87 \%$. Conclusion: Multiparametric 3T-MRI breast imaging shows excellent results in evaluation of breast cancer compared to histological findings, both for primary tumor and nodal metastases. The greatest contribution to improving diagnostic performance is the evaluation of diffusion.
\end{abstract}

Correspondence to: Hynek Mírka, MD, Ph.D., Department of Radiology, Medical School and Teaching Hospital Pilsen, Charles University in Prague, Alej Svobody 80, 30460 Plzen, Czech Republic. Tel: +420 377103456, Fax: +420 377103438, e-mail: mirka@fnplzen.cz

Key Words: Breast cancer, magnetic resonance imaging, multiparametric imaging, diffudion weighted imaging, magnetic resonance spectroscopy, dynamic contrast enhanced magnetic resonance.
Magnetic resonance imaging (MRI) is the imaging modality of choice for advanced assessment of changes in the mammary gland to confirm benignity or malignancy of the lesion and for precise staging before surgery, in particular to confirm or rule out multifocal and multilateral carcinoma (1, 2). Although targeted MRI of the breast is well-established in clinical practice, the introduction of 3T scanners into clinical routine use, recent advances in receiving coil design, and new scanning sequences has brought new options for multiparametric high-resolution breast imaging. Conventional protocols combining morphological T1-weighted images, T2weighted images including T2 short tau inversion recovery (STIR) sequences and finally dynamic T1-weighted gradient echo images after administration of gadolinium contrast media are routinely used today. New techniques, widely used in other indications for tumor imaging, diffusion-weighted imaging (DWI), ${ }^{1} \mathrm{H}$ MR spectroscopy (MRS) and pharmacokinetic analysis methods, have minimally been used for breast imaging. The aim of this study was to evaluate our experience with multiparametric breast imaging using a 3T-MRI scanner with dedicated 18-channel coil compared to histological findings in women after surgery. At the same time, we evaluated the benefits of the individual parameters for the detection of breast carcinoma.

\section{Materials and Methods}

The study included 100 women (aged 23 to 71 years, mean age $=52.7$ years) with Breast Imaging Reporting and Data System (Bi-RADS) 4 to 6 lesions in mammography who were examined on 3T-MRI and subsequently underwent surgery. Five women had surgery on both breasts. All patients underwent a simultaneous multiparametric breast examination on 3T-MRI (Skyra, Siemens Healthcare, Erlangen, Germany) using an 18-channel dedicated coil (Breast 18, Siemens Healthcare, Erlangen, Germany). The imaging protocol included T1-weighted images, T2-weighted images with suppression of the signal from adipose tissue by STIR, DWI acquired by echo planar sequences with suppression of the signal from adipose tissue by spectrally adiabatic inversion recovery (SPAIR), including the calculation of the apparent diffusion coefficient (ADC) map. All images were made in the transverse 
plane. The protocol continued with dynamic gradient echo T1weighted images (DynaViews) with suppression of the signal from adipose tissue by the spectral attenuated inversion recovery (SPAIR) technique after administration of contrast agent in six cycles, where the contrast agent gadoterate meglumine (Dotarem, Guerbet, Paris, France; relaxivity $r_{1}=3.4 \pm 0.4 \mathrm{mmol} / \mathrm{s}$ at $3 \mathrm{~T}$ ) was administered at 0.1 $\mathrm{mmol} / \mathrm{kg}$ between the first and second acquisition, and image subtractions and whole-volume breast reconstructions were also made in-line using the maximum intensity projection (MIP) algorithm in the transverse and coronal planes. After the evaluation of the post-contrast phase, lesions were selected and spectroscopic measurements were performed by single voxel spectroscopy (SVS) using the turbo gradient-spin-echo (GRASE) sequence with selective pulse suppression of water and lipid signals.

The findings were evaluated using the double reading method. Both evaluations were performed by radiologists with 20 years of experience in breast MRI. The shape and internal structure of the lesion were evaluated. In DWI, the ADC maps were evaluated, and signs of diffusion restriction were monitored in relation to the focal lesions enhanced after the administration of the contrast agent, significant decrease of ADC value suspected from carcinoma was set below the level of $1,000 \times 10^{-6} \mathrm{~mm}^{2} / \mathrm{s}$. Visual evaluation of the metabolite spectra was performed as part of the assessment, with a special focus on choline, using the total-choline-peak (tpCho) method and evaluation of the tpCho peak integral and the level of the arbitrary value (AU) significant for malignancy was set to 7 AU. An essential part of the evaluation was the use of pharmacokinetic analysis (Tissue4D; Siemens Healthcare).

Breast surgery, i.e. partial resection or subcutaneous or total mastectomy, was performed in all patients, while patients with malignant findings underwent resection of axillary lymph nodes based on the results of a sentinel node biopsy or axillary lymph node dissection in the case of severe preoperative findings. All resected tissue samples were subjected to histopathological examination, including an evaluation of structure, nuclear grading, proliferation activity, presence of estrogen and progesterone receptors, and the presence of human epidermal growth factor receptor 2 neu (HER2/neu) gene mutation.

The findings obtained by pathologist were compared with preoperative findings on MRI, and sensitivity and specificity was evaluated in the entire population and for the individual findings.

All imaging and surgical procedures were performed after informed consent was obtained, the study was carried out in accordance with Helsinki declaration.

\section{Results}

There were 105 surgeries to be compared with results of breast MRI. A total of 76 malignant findings were detected in the group of breasts operated on, and benign lesions were found in 29 breasts (Table I). In 76 surgeries, lymph nodes were removed from the axilla, and metastatic involvement was confirmed by a pathological anatomist in six cases.

When comparing MRI results with pathological and anatomical findings, a sensitivity of $98.68 \%(75 / 76)$ was found throughout the population, with specificity of $86.20 \%(25 / 29)$, when all evaluation parameters were considered, such as lesion shape, the presence of diffusion restriction, increased choline in the spectrum and enhancement of the lesion. Four false-
Table I. Summary of diagnoses proven by biopsy.

\begin{tabular}{lr}
\hline Diagnosis & $\mathrm{N}$ \\
\hline Invasive ductal carcinoma & 38 \\
Invasive lobular carcinoma & 31 \\
Ductal carcinoma in situ & 7 \\
Sclerosing adenosis & 11 \\
Papilloma/papillomatosis & 8 \\
Fibroadenoma & 7 \\
Abscess & 2 \\
Granuloma & 1 \\
Total & 105 \\
\hline
\end{tabular}

positive results were detected in the population, which were confirmed as ductal fibroadenoma in one case by histology and showed enhancement characteristics corresponding to rapid enhancement, slow elution of the contrast medium and an increased percentage of choline in the spectrum, on the other hand, its shape was round and its diffusivity was relatively high, higher than that of the pectoralis major muscle; with additional false-positive findings confirmed as two cases of sclerosing adenosis and one case of papilloma.

In a more detailed analysis of the sensitivity and specificity of signs for breast cancer detection in the study population, the most valuable findings were diffusion restriction with the highest sensitivity and specificity: sensitivity of $90.79 \%(69 / 76)$, specificity of $89.66 \%(26 / 29)$, and increased choline in the spectrum above $7 \mathrm{AU}$ when assessing the tpCho integral, with sensitivity of $68.42 \%$ (52/76) and specificity of $93.10 \%$ (27/29).

Traditional evaluation of the enhancement curve characteristics was affected by a very low sensitivity $(45.05 \%, 35 / 76)$ with a relatively high specificity $(72.41 \%$, 21/29) (Tables II and III). Subjective criteria were used to evaluate the lymph nodes based on the shape and size of the nodes and the character of their enhancement. Eeven for precise assessment in multiple lymph node involvement, and in solitary nodal metastases the results were very good, with satisfactory values of sensitivity $(92.59 \%, 25 / 27)$ and specificity $(93.87 \%, 46 / 49)$ being achieved in the entire population of women who underwent surgeries including axillary lymph node removal (Table IV).

\section{Discussion}

Non-contrast-enhanced morphological imaging based on T1and T2-weighted images have only limited value in detection and characterization of breast carcinoma. We use T1 fast lowangle single shot (FLASH) for the detection of adipose tissue in the lesions, and in cases after biopsy, for the detection of hemoglobin degradation products, especially methemoglobin 
Table II. Results of magnetic resonance imaging versus biopsy findings.

\begin{tabular}{|c|c|c|c|c|c|c|}
\hline & $\begin{array}{c}\text { Multi- } \\
\text { parametric } \\
\text { assessment }\end{array}$ & $\begin{array}{l}\text { Spiculated or } \\
\text { irregular } \\
\text { lesion margins }\end{array}$ & $\begin{array}{c}\text { ADC value } \\
<1000 \times 10^{-6} \\
\mathrm{~mm}^{2} / \mathrm{s}\end{array}$ & $\begin{array}{l}\text { tpCho } \\
\text { integral } \\
>7 \mathrm{AU}\end{array}$ & $\begin{array}{c}\text { Enhancement } \\
\text { curve wash } \\
\text { out or plateau }\end{array}$ & $\begin{array}{c}\text { Enhancement } \\
\text { curve progressive } \\
\text { or logarithmic }\end{array}$ \\
\hline True positive, $\mathrm{n}$ & 75 & 46 & 69 & 52 & 35 & 41 \\
\hline False negative, $\mathrm{n}$ & 1 & 30 & 7 & 24 & 41 & 35 \\
\hline True negative, $\mathrm{n}$ & 25 & 17 & 26 & 27 & 21 & 8 \\
\hline False positive, $\mathrm{n}$ & 4 & 12 & 3 & 2 & 8 & 21 \\
\hline Total, $\mathrm{n}$ & 105 & 105 & 105 & 105 & 105 & 105 \\
\hline Sensitivity & $98.68 \%$ & $60.53 \%$ & $90.79 \%$ & $68.42 \%$ & $46.05 \%$ & $53.95 \%$ \\
\hline Specificity & $86.21 \%$ & $58.62 \%$ & $89.66 \%$ & $93.10 \%$ & $72.41 \%$ & $27.59 \%$ \\
\hline Positive predictive value & $94.94 \%$ & $79.31 \%$ & $95.83 \%$ & $96.30 \%$ & $81.40 \%$ & $66.13 \%$ \\
\hline Negative predictive value & $96.15 \%$ & $36.17 \%$ & $78.79 \%$ & $52.94 \%$ & $33.87 \%$ & $18.60 \%$ \\
\hline
\end{tabular}

ADC: Apparent difusion coefficient; AU: arbitrary unit; tpCho: total-choline-peak.

(1). T2-Weighted images are aimed at imaging tissues with relatively high or very high water content such as cysts or fluid collections in abscesses. When evaluating the shape of a pathological lesion, post-contrast T1-weighted images with adipose tissue signal suppression are of the utmost importance, as they allow better differentiation between smooth oval, lobular and stellar lesions (2). When using isotropic or near isotropic imaging, the shape of the lesion can be evaluated in any plane by multiplanar reconstruction.

DWI in breast diseases is useful for assessing tissue microstructure. Highly cellular tumors with small cells and small intercellular space lead to a limitation of Brown's free movement of water molecules, resulting in diffusion restriction (3-5). However, the character of malignant tumor growth may differ. In particular, higher grade tumors exhibit the highest degree of diffusion restriction compared to lower grade tumors. For tumors of grade 3 or more, the ADC is about $1,000 \times 10^{-6} \mathrm{~mm} / \mathrm{s}^{2}$ or even lower (4-7). If an ADC value of $1,270 \times 10^{-6} \mathrm{~mm} / \mathrm{s} 2$ or lower is chosen for the detection of a malignant lesion in the breast, the sensitivity of carcinoma detection is $100 \%$ and the positive predictive value is $65 \%$. With decreasing $\mathrm{ADC}$ value, sensitivity decreases (well-differentiated carcinomas types are falsely negative), but positive predictive value increases. In contrast, for benign lesions, the ADC values are above $1,500 \times 10^{-6}$ $\mathrm{mm} / \mathrm{s}^{2}$ up to $2,000 \times 10^{-6} \mathrm{~mm} / \mathrm{s}^{2}(6)$.

Dynamic post-contrast imaging has been used in the diagnosis of breast cancer by MRI since the early 1990s. At present, high spatial resolution techniques with voxel size of $1 \times 1 \mathrm{~mm}$ are preferred as they allow detection of small lesions of less than $5 \mathrm{~mm}$ (1). Conventional imaging algorithms used data acquisition at $1 \mathrm{~min}$. intervals of up to 9 to 10 minutes after contrast agent administration. In our practice, we used five post-contrast series to calculate the enhancement curve and also to evaluate the pharmacokinetic parameters. When
Table III. Enhancement curves in invasive ductal carcinoma and invasive lobular carcinoma.

\begin{tabular}{lcc}
\hline & \multicolumn{2}{c}{ Enhancement curve wash out or plateau } \\
\cline { 2 - 3 } Finding & IDC & ILC \\
\hline True positive, $\mathrm{n}$ & 26 & 10 \\
False negative, $\mathrm{n}$ & 12 & 21 \\
Total & 38 & 31 \\
Sensitivity* & $68.42 \%$ & $32.26 \%$ \\
\hline
\end{tabular}

IDC: Invasive ductal carcinoma; ILC: invasive lobular carcinoma. *Specificity and other parameters cannot be calculated.

Table IV. Axillary lymph node assessment: magnetic resonance imaging versus biopsy findings.

\begin{tabular}{lc}
\hline Finding & $\mathrm{N}$ \\
\hline True positive & 26 \\
False negative & 2 \\
True negative & 46 \\
False positive & 2 \\
Total & 76 \\
Sensitivity & $92.86 \%$ \\
Specificity & $95.83 \%$ \\
Positive predictive value & $92.86 \%$ \\
Negative predictive value & $95.83 \%$ \\
\hline
\end{tabular}

evaluating the findings, it is important to assess the initial skewness of the curve and its subsequent development. For carcinoma, a typical tissue intensity curve is traditionally rapid rising with a subsequent decrease in intensity (washout) (2), while a slow continuous increase in tissue intensity resembling 


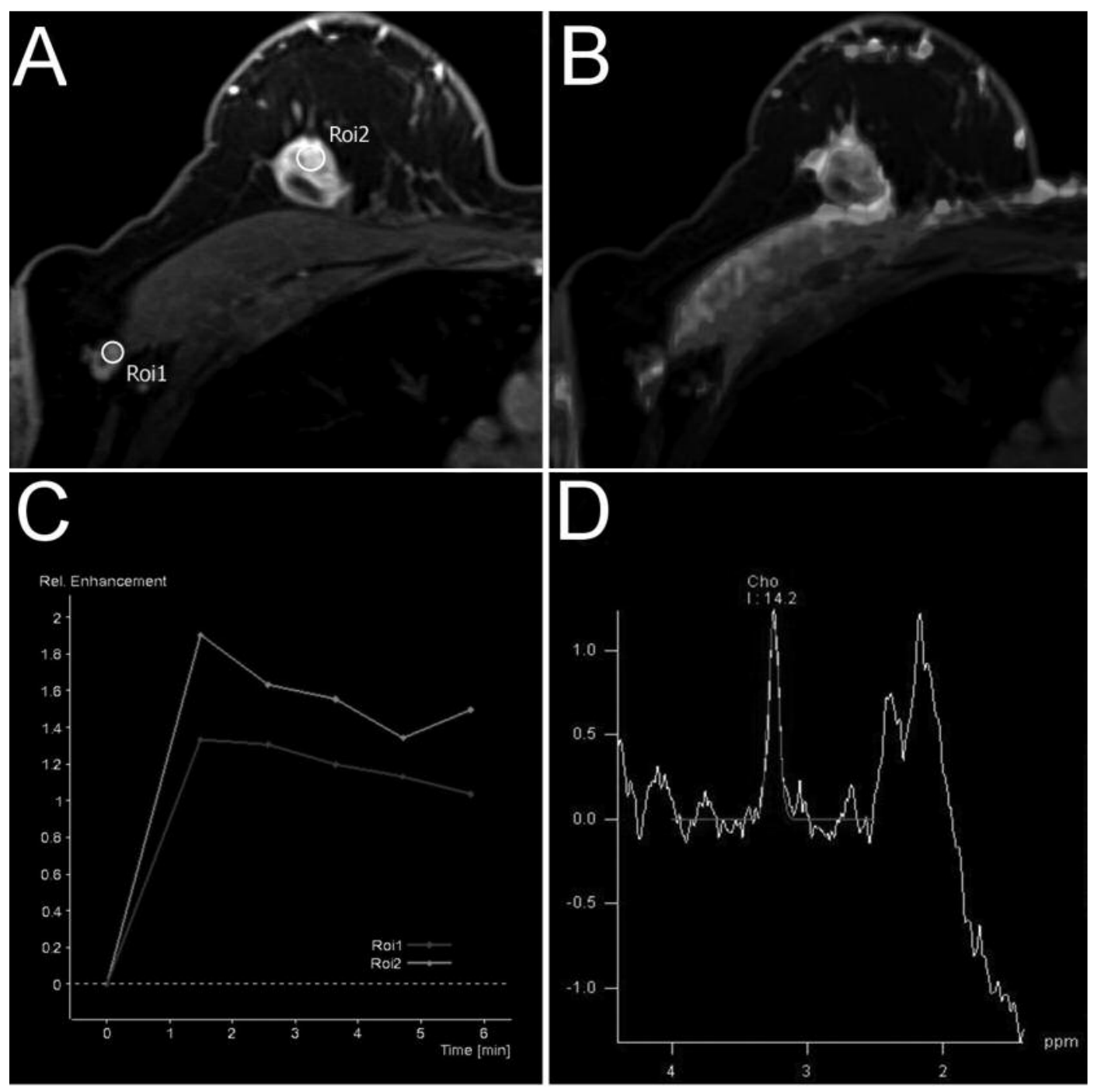

Figure 1. Ductal invasive carcinoma with activated (benign) axillary lymph node. A: T1-Weighted postcontrast image (Roil: lymph node, Roi2: carcinoma). B: Diffusion-weighted image showing restriction of diffusion within the tumor. C: Enhancement curves for the lymph node (Roil) and tumorous tissue (Roi2). D: Magnetic resonance imaging spectroscopy shows high choline (Cho) peak integral.

the shape of a logarithmic curve is typical for benign lesions. However, our results show that the traditional 'malignant' curve is relatively rare for lobular carcinomas, although it describes slightly more than half of ductal carcinomas cases (Figure 1). For these reasons, an evaluation based on the enhancement curve alone is not sufficient. Finding of a 'benign' curve in malignant tumors might be explained by desmoplasia, or infiltrative growth, which may be the cause of relatively low vascularity (Figure 2). Overall, it can be stated that the character of breast enhancement is relatively variable and should always be evaluated in the wider context of other findings. The sensitivity of dynamic post-contrast imaging usually exceeds $90 \%$ in expansive tumors but the specificity varies from $37 \%$ to $95 \%$ (1). The values of these parameters are significantly lower for infiltrative tumors. The relative larger count for lobular carcinoma and ductal carcinoma in situ leads to lower sensitivity and specificity for detection of carcinoma using enhancement alone.

Examination of breast tissue is a relatively new use of MRS. When assessing focal breast lesions, the method is based on the presence of choline derivatives, as with other tissues. Their resonance appears at around $3.2 \mathrm{ppm} \mathrm{(8).} \mathrm{Choline,}$ phosphocholine, glycerophosphocholine and taurine, substances involved in the building and destruction of biomembranes composed of the phospholipid bilayer, are believed to contribute to the resonance signal. Choline oscillation is complex in breast tissue and is termed tpCho. SVS is more commonly used than chemical shift imaging (CSI). The voxel volume is customized 

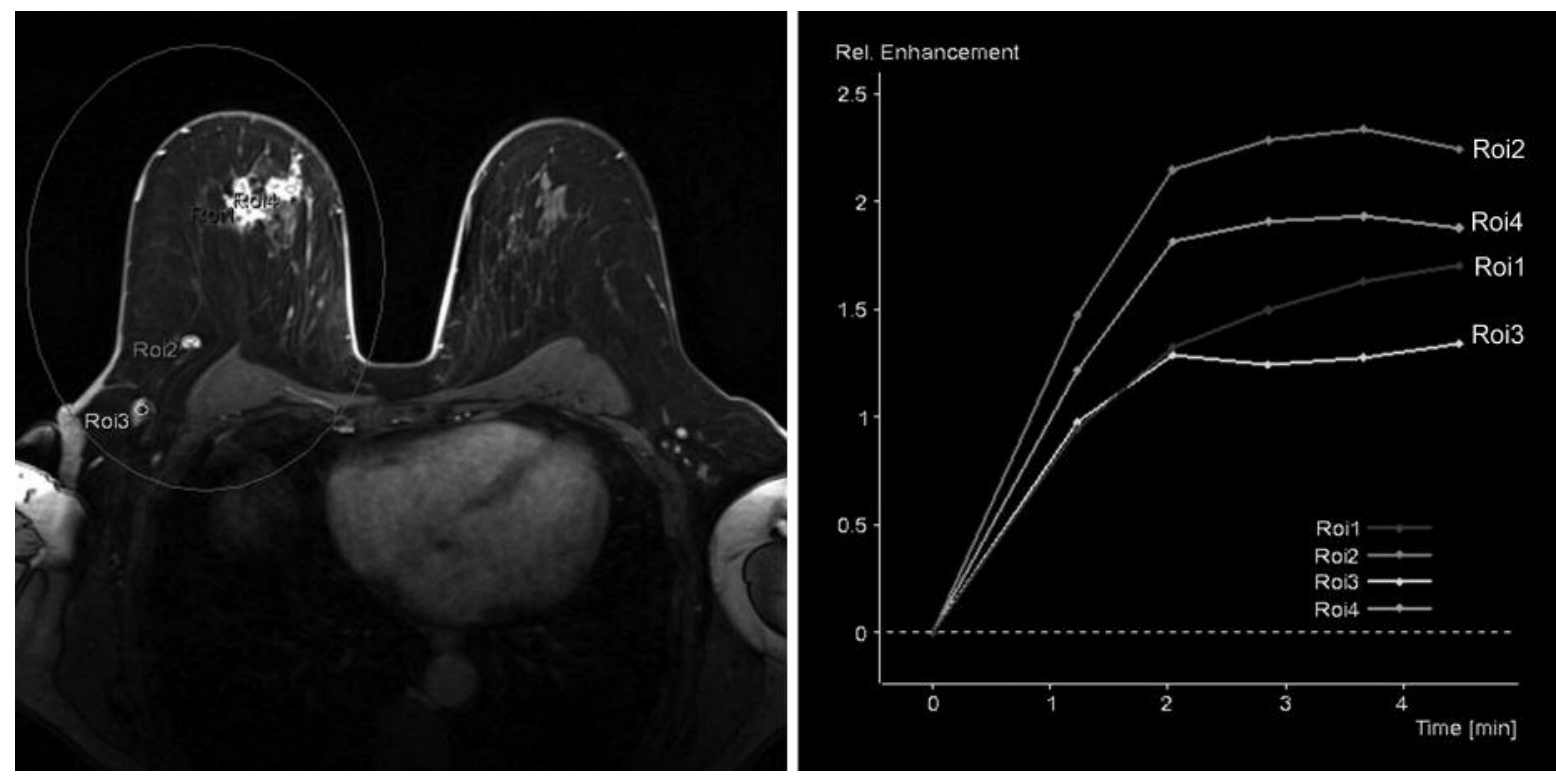

Figure 2. A: Lobular invasive carcinoma (Roil and Roi4) with metastases to the axillary lymph nodes (Roi2 and Roi3). B: Note the logarithmic shape of the enhancement curves.

to the volume of the lesion, and the quality of the obtained spectrum is higher. There are relatively few studies using CSI, but these show results similar to those with SVS. While values of the tpCho integral greater than 7 were considered pathological here, we nevertheless achieved similar sensitivity as when the cut-off level was 2.6 (9). In other comparable studies, higher choline levels were found in some fibroadenomas, similar to the results of our study, as opposed to benign lesions, such as inflammation or sclerosing adenosis. These lesions have been found to have low integral values, with a median of about 2 . However, values above 4.5 have not been found in fibroadenomas in published reports, but in our population fibroadenoma (false-positive finding) had an integral value of 8.02 . The value of the tpCho integral has a marked relationship with the differentiation of lesions and increases in dedifferentiated carcinoma types, with high proliferative activity, and then reaches a significantly higher value than for well-differentiated lesions, both in lobular and ductal carcinomas $(8,9)$. In contrast, in tumors with very low proliferative activity, especially lobular, the choline fraction in the spectrum is scarce in lesions with a benign character. Spectroscopy, therefore, has a significant additive role in the detection of carcinoma but also is of great importance in assessing the aggressiveness and biological behavior of the carcinoma.

Our patient sample was affected by an imbalance in the frequency of lobular carcinomas in reality, the reason being that MRI is more often indicated for biopsy-confirmed lobular carcinomas due to the more probable presence of multifocality and bilaterality. We achieved very satisfactory results by combining examinations of multiple parameters. DWI and spectroscopy should be considered as the most valuable in the evaluation of lesions, while dynamic imaging appears to be of less importance for the differentiation of benign and malignant lesions. However, it is a necessary part of the examination, which is used to target spectroscopic imaging and other methods of targeted evaluation. One way of the future development of breast imaging could be positron-emission tomography-MRI, which will further improvement of multiparametric diagnostics by including energy metabolism $(13,14)$.

\section{Conclusion}

Multiparametric 3T-MRI breast imaging shows excellent results in breast cancer evaluation compared to histological findings, both for primary tumor and nodal metastases. The greatest contribution to improving diagnostic performance is the evaluation of water molecule diffusion, where the T2weighting nature exploits the greatest benefits of 3T-MRI, despite distortion problems.

\section{Acknowledgements}

This study was supported by the project of the Ministry of Health Conceptual Development of Research Institutions 00669806 - FN Plzen, by the project of the Charles University Prague Progress Q39 and by the project CZ.1.05/2.1.00/03.0076 from European Regional Development Fund. 


\section{References}

1 Kuhl CK: Breast MR imaging at 3T. Magn Reson Imaging Clin N Am 15(3): 315-320, 2007.

2 Lehotska V, Rauova K and Vanovcanova L: MR-mammography - impact on disease extent determination and surgical treatment of invasive ductal and lobular breast cancers. Neoplasma 62(2): 269-277, 2015.

3 Al Rashidi N, Waiter G, Redpath T and Gilbert FJ: Assessment of the apparent diffusion coefficient (ADC) of normal breast tissue during the menstrual cycle at $3 \mathrm{~T}$ using image segmentation. Eur J Radiol 81(Suppl 1): S1-3, 2012.

4 Cakir O, Arslan A, Inan N, Anık Y, Sarısoy T, Gumustas S and Akansel G: Comparison of the diagnostic performances of diffusion parameters in diffusion weighted imaging and diffusion tensor imaging of breast lesions. Eur J Radiol 82(12): e801-806, 2013.

5 De Felice C, Cipolla V, Guerrieri D, Santucci D, Musella A, Porfiri LM and Meggiorini ML: Apparent diffusion coefficient on 3.0 Tesla magnetic resonance imaging and prognostic factors in breast cancer. Eur J Gynaecol Oncol 35(4): 408-414, 2014.

6 Cipolla V, Santucci D, Guerrieri D, Chen J, Guan W, Wang H, Yu H, Liu S, Zhou Z, Yang X and Liu T: Correlation between 3T apparent diffusion coefficient values and grading of invasive breast carcinoma. Eur J Radiol 83(12): 2144-2150, 2014.

7 Wisner DJ, Rogers N, Deshpande VS, Newitt DN, Laub GA, Porter DA, Kornak J, Joe BN and Hylton NM: High-resolution diffusion-weighted imaging for the separation of benign from malignant BI-RADS 4/5 lesions found on breast MRI at 3T. J Magn Reson Imaging 40(3): 674-681, 2014.

8 Battal B, Akgun V and Karaman B: Value of $3 \mathrm{~T}{ }^{1} \mathrm{H}$-magnetic resonance spectroscopy in the differentiation of benign and malignant breast tumors. Acta Radiol 55(4): 416-417, 2014.
9 Gruber S, Debski BK, Pinker K, Chmelik M, Grabner G, Helbich T, Trattnig S and Bogner W: Three-dimensional proton MR spectroscopic imaging at $3 \mathrm{~T}$ for the differentiation of benign and malignant breast lesions. Radiology 261(3): 752-761, 2011.

10 Le Y, Kipfer H, Majidi S, Holz S, Dale B, Geppert C, Kroeker $\mathrm{R}$ and Lin $\mathrm{C}$ : Application of time-resolved angiography with stochastic trajectories (TWIST)-Dixon in dynamic contrastenhanced (DCE) breast MRI. J Magn Reson Imaging 38(5): 1033-1042, 2013.

11 Lourenco AP, Donegan L, Khalil $\mathrm{H}$ and Mainiero MB: Improving outcomes of screening breast MRI with practice evolution: initial clinical experience with $3 \mathrm{~T}$ compared to $1.5 \mathrm{~T}$. J Magn Reson Imaging 39(3): 535-539, 2014.

12 Rahbar H, DeMartini WB, Lee AY, Partridge SC, Peacock S and Lehman CD: Accuracy of 3T versus $1.5 \mathrm{~T}$ breast MRI for preoperative assessment of extent of disease in newly diagnosed DCIS. Eur J Radiol 84(4): 611-616, 2015.

13 Pinker K, Bogner W, Baltzer P, Karanikas G, Magometschnigg H, Brader P, Gruber S, Bickel H, Dubsky P, Bago-Horvath Z, Bartsch R, Weber M, Trattnig S and Helbich TH: Improved differentiation of benign and malignant breast tumors with multiparametric 18fluorodeoxyglucose positron emission tomography magnetic resonance imaging: a feasibility study. Clin Cancer Res 20(13): 3540-3549, 2014.

14 Taneja S, Jena A, Goel R, Sarin R and Kaul S: Simultaneous whole-body ${ }^{18} \mathrm{~F}-\mathrm{FDG}$ PET-MRI in primary staging of breast cancer: a pilot study. Eur J Radiol 83(12): 2231-2239, 2014.

Received September 6, 2017 Revised September 25, 2017 Accepted September 27, 2017 